\title{
The Diversity of Low-mass Exoplanets Characterized via Transit Timing
}

\author{
Daniel Jontof-Hutter ${ }^{1}$, Eric B. Ford ${ }^{1}$, Jason F. Rowe ${ }^{2}$, \\ Jack. J. Lissauer ${ }^{3}$ and Daniel C. Fabrycky ${ }^{4}$ \\ ${ }^{1}$ Dept. of Astronomy, Pennsylvania State University, \\ University Park, PA-16802, United States \\ email: dxj14@psu.edu \\ ${ }^{2}$ Université de Montréal \\ ${ }^{3}$ NASA Ames Research Center \\ ${ }^{4}$ University of Chicago
}

\begin{abstract}
Transit timing variations (TTV) in multi-transiting systems enables precise characterizations of low-mass planets and their orbits. The range of orbital periods and incident fluxes with detailed TTV constraints complements the radial velocity sample for low-mass planets, pushing exoplanet characterization to the regime sub-Earth size planets and out to Mercurylike distances. This has revealed an astonishing diversity in the density of super-Earth mass planets. We summarize these and other contributions to exoplanet science from TTVs.
\end{abstract}

Keywords. Dynamics, Exoplanets, Multi-planet systems

\section{Introduction}

The Kepler mission's discovery of thousands of planet candidates within multi-transiting planetary systems was unanticipated (Borucki et al. 2008), although their value in causing detectable transit timing variations (TTVs) was predicted (Holman \& Murray 2005; Agol et al. 2005). The first detection of TTVs at Kepler-9 indicated that radial velocity confirmations (RV) would be complemented by TTVs, using just the Kepler time series photometry (Holman et al. 2010).

The confirmation of many planet candidates with TTVs soon followed (Ford et al. 2011, 2012a; Steffen et al. 2012a). This was possible because the detection of anti-correlated TTV signals is difficult to attribute to anything other than two interacting planets orbiting the same star. The rapid confirmation of the multi-transiting planet systems with TTVs has greatly enhanced the return of the Kepler mission. In some cases, even the absence of a TTV signal has enabled planet candidates to be validated where low mass stellar companions or brown dwarfs would induce detectable TTVs (Lissauer et al. 2014; Rowe et al. 2014).

Using just the light curves, these confirmations were possible far quicker than could be expected of ground-based radial velocity follow-up (Fabrycky et al. 2012; Ford et al. 2012b; Steffen et al. 2013). Furthermore, detailed studies of individual systems including Kepler-11 (Lissauer et al. 2011a) and Kepler-30 (Sanchis-Ojeda et al. 2012) permitted the characterization of low-mass planets around faint targets. Characterization via transit timing has benefited not only from the abundance of compact multi-planet systems, but also the abundance near mean motion resonances (Lissauer et al. 2011b; Lithwick \& Wu 2012; Fabrycky et al. 2014), which causes coherent perturbations and strong TTV signals. 
In parallel to these data-driven developments, the progress in analytical models to explain the TTVs has continued. Lithwick et al. (2012) developed a solution for TTVs for planet pairs near first-order mean motion resonances. This solution enabled initial mass estimates and for dozens of planet pairs near first-order resonances (Wu \& Lithwick 2013; Xie 2013; Hadden \& Lithwick 2014).

In the absence of detectable frequencies in addition to the near resonance terms (Nesvorný \& Morbidelli 2008), the solution of Lithwick et al. (2012) highlights the degeneracy between dynamical masses and the relative free eccentricities between interacting planets, but also enabled an initial characterization of the distribution of orbital eccentricities among Kepler's multi-planet systems. Both the sample of TTV detections and the distribution of transit duration ratios indicate a low eccentricity dispersion among Kepler's multis (Hadden \& Lithwick 2014; Fabrycky et al. 2014).

The transit durations also imply a low inclination dispersion (Fabrycky et al. 2014). While strong TTV signals can detect moderate inclinations (Nesvorný et al. 2013), among low-mass planets TTVs are insensitive to mutual inclinations to first order (Nesvorný \& Vokrouhlický 2014). While this has made it justifiable to reduce the number of free parameters in characterizing TTV systems, the price is that the light curve gives information on significant mutual inclinations only in rare cases, where signals are very strong or where planet-planet eclipses are detected during transit (Ragozzine \& Holman 2010; Masuda et al. 2013).

In addition to the coherent TTV signal caused by a near resonant orbital period ratio, the effect of each conjunction at the synodic frequency causes so-called "chopping" in the TTV signal, breaking the mass-eccentricity in cases of high signal TTVs. In such cases, there are analytical solutions correct to first-order in eccentricity not only near mean motion resonances (Deck \& Agol 2015; Agol \& Deck 2015).

\section{Low-mass transiting planets characterized via RV and TTV}

Figure 1 highlights how TTVs complement the RV sample. Neither RV nor TTV sample the entire range of Kepler's period-radius distribution, because of the detection biases in both techniques. For super-Earth mass planets, most RV detections have orbital periods of a few days or less. TTV signals, on the other hand, scale with orbital period, and detections begin at a few days. In the Kepler sample, the maximum orbital period for planets with detected TTVs is limited only by the 4 year photometric baseline. Kepler$87 \mathrm{c}$ with an orbital period of 191 days, has the longest orbital period with a measured mass from TTVs (Ofir et al. 2014). This is one of several TTV systems pushing low-mass exoplanet characterization beyond Mercury-like distances.

TTVs have also characterized the lowest-mass transiting exoplanets, including the first sub-Earth sized planet with a measured density (Jontof-Hutter et al. 2015). Kepler-138 $\mathrm{b}$ has a size and mass consistent with Mars, and is the innermost of three transiting exoplanets orbiting an M-dwarf. With orbital periods at $\sim 10$ days, $\sim 14$ days, Kepler$138 \mathrm{~b}\left(0.5 \mathrm{R}_{\oplus}\right.$ in size) and Kepler-138 c $\left(1.2 \mathrm{R}_{\oplus}\right)$ orbit near the $4: 3$ (first-order) mean motion resonance, and at $\sim 23$ days, Kepler-138 d $\left(1.2 \mathrm{R}_{\oplus}\right)$ is near the $5: 3$ (second-order) mean motion resonance with Kepler-138 c. All three planets show TTVs consistent with the periods expected of their near resonant orbital periods. The TTVs and a sample of dynamical fits to the TTVs are shown in Figure 2.

Kipping et al. (2014) analyzed the TTVs of the outer pair and found that the outer two planets have mass ratio of $\sim 3$. Using the complete 17 quarters of Kepler data and a three-planet model to the TTVs, Jontof-Hutter et al. (2015) found a similar mass ratio between the outer pair. The middle planet has a likely rocky composition, while the 


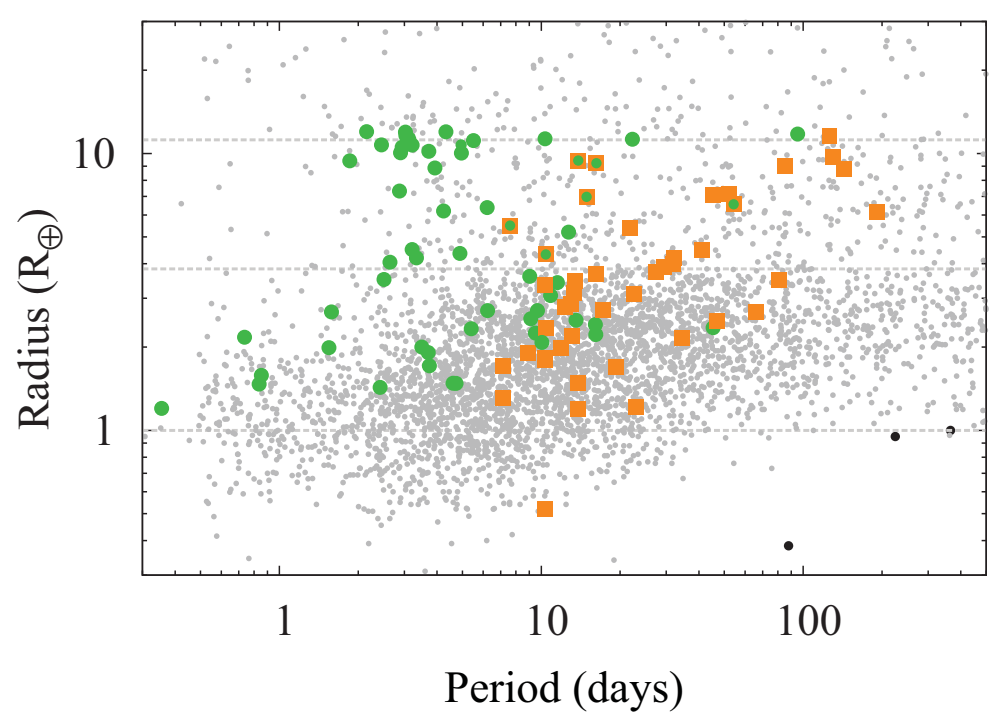

Figure 1. Kepler's period-radius distribution. The grey points are the complete sample of planet candidates without uncertainties. The green circles are the subsample with published radial velocity detections, and orange squares denote the TTV sample with detailed dynamical fits. Some transiting planets have both RV and TTV data.

outer planet's composition is consistent with a mixture of rock and hydrogen or rock and water.

Kepler-138 c and Kepler-138 d are typical of the wide range in density seen in superEarths, as shown in Figure 3. Kepler-78 b, characterized by RV, has a similar size and mass to Kepler-138 c (Howard et al. 2013; Pepe et al. 2013). These characterizations probe the boundary between rocky planets and super-Earth mass planets that retain deep atmospheres.

Dressing et al. (2015) show evidence of a pile-up at rock-like bulk densities up to $1.6 R_{\oplus}$ among the strongest mass detections including Kepler-93 b, Kepler-36 b (Carter et al. 2012), CoRoT-7 b (Haywood et al. 2014), 55 Cnc e (Gillon et al. 2012), Kepler10b (Batalha et al. 2011), Kepler-99 b and Kepler-406 b (Marcy et al. 2014). However, strong mass upper limits on Kepler-11 b, Kepler-11 f (Lissauer et al. 2013), Kepler-20 b (Gautier et al. 2012) and Kepler-231 c (Kipping et al. 2014) imply that low densities, and therefore significant volumes of volatiles is possible among planets larger than $1.6 R_{\oplus}$. This is consistent with the findings of Rogers (2015), that the majority of planets larger than $1.6 R_{\oplus}$ are not primarily rocky by volume.

\section{RV and TTVs: a complementary sample of mass and composition}

Most of the known low-density super-Earth mass planets have been characterized from TTV analysis, including all six planets known to orbit Kepler-11 (Lissauer et al. 2011a, 2013). The volumes of these planets can be explained with small mass fractions in a deep atmosphere of $\mathrm{H} / \mathrm{He}$. However, the measured masses from TTVs of Kepler-79 d (Jontof-Hutter et al. 2014), Kepler-51 b-d (Masuda 2014) and Kepler-87 c (Ofir et al. 2014) (all less than $10 M_{\oplus}$, despite sizes larger than $6 R_{\oplus}$ ) require significant fractions of their mass in their atmospheres. These planets reveal extraordinary variety in the bulk 

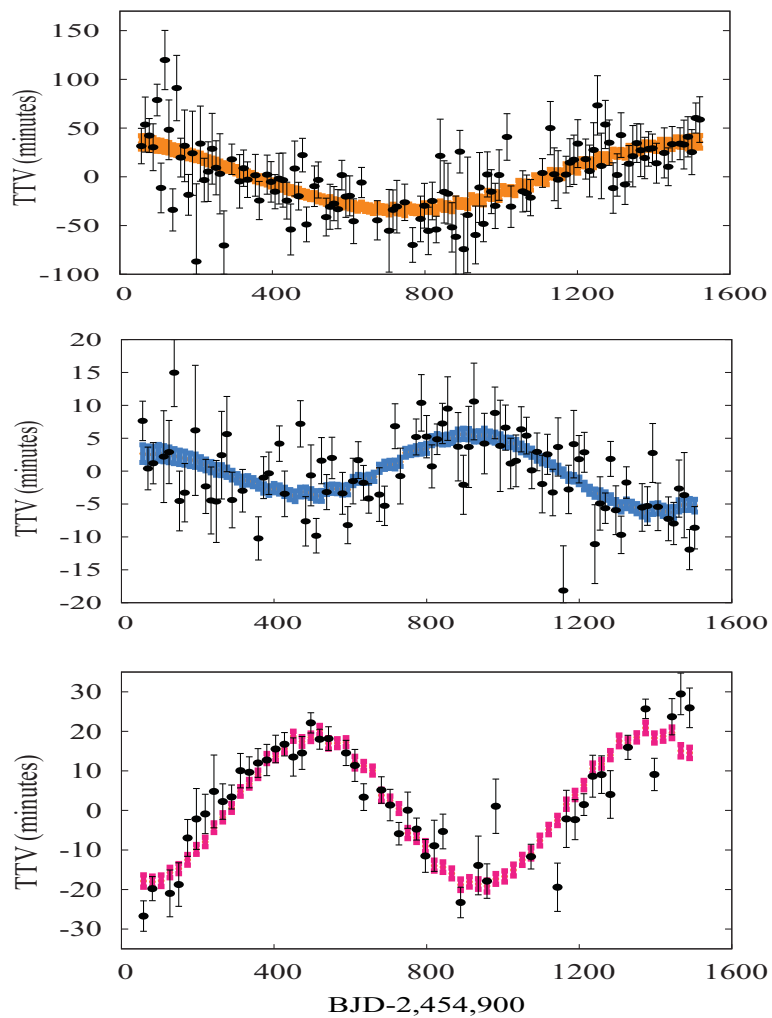

Figure 2. Model fits to the transit times of all transiting planets at Kepler-138. The black points are the measured differences between each transit time and a calculated linear fit to the transit times. In color are the $1 \sigma$ range of simulated transit times from posterior samples of our model parameters. (Adapted from Jontof-Hutter et al. 2015)

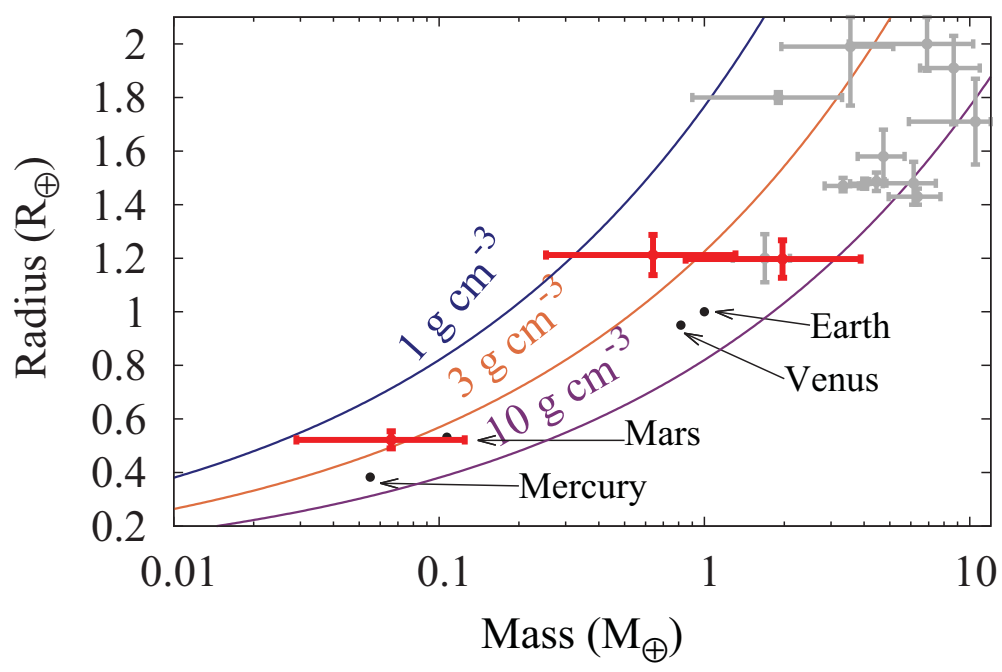

Figure 3. Mass-radius diagram of planets smaller than $2.1 R_{\oplus}$. In red are the three transiting planets of Kepler-138, compared to Mercury, Venus, Earth and Mars (black points). In grey are other well-characterized exoplanets. The colored curves mark contours of constant bulk density, at $1 \mathrm{~g} \mathrm{~cm}^{-3}, 3 \mathrm{~g} \mathrm{~cm}^{-3}$ and $10 \mathrm{~g} \mathrm{~cm}^{-3}$. 


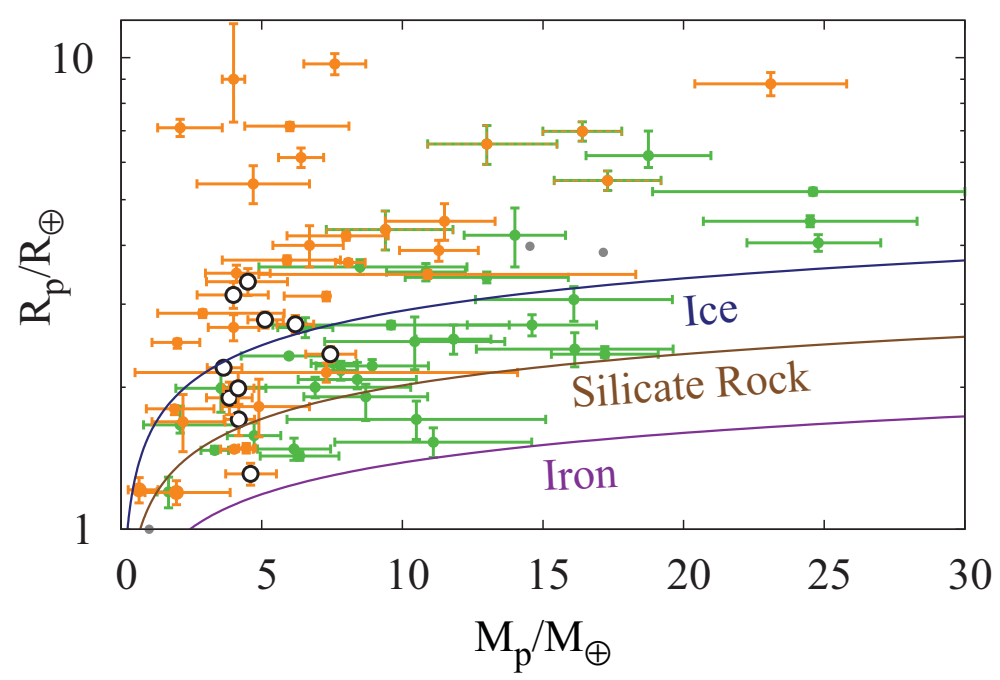

Figure 4. Mass-radius diagram of planets less than $25 M_{\oplus}$. The grey points are planets of the Solar System. In green are planets characterized via RV, and in orange are the TTV sample. Planets shown in orange and green are constrained by RV and TTV data. The colored curves mark solutions for planets of pure iron, silicate rock, or water (Fortney et al. 2007). The open circles mark ten new additions to the mass radius diagram with TTVs that confirm the diverse range in density in the 3-8 $M_{\oplus}$ mass range.

density of super-Earth mass planets. At $6 M_{\oplus}$ and $7 R_{\oplus}$, Kepler-79 d has a bulk density below $0.1 \mathrm{~g} \mathrm{~cm}^{-3}$, and is likely significantly more than $10 \% \mathrm{H} / \mathrm{He}$ by mass. All three planets transiting Kepler-51 are even less dense.

Figure 4 shows the mass-radius diagram for low-mass planets, highlighting the division between the RV and TTV sample, including six unpublished additions (Jontof-Hutter et al. 2015). While there is some overlap where there is both RV and TTV data- including Kepler-18 (Cochran et al. 2011) and Kepler-89 (Weiss et al. 2013; Masuda et al. 2013), the TTV planets appear systematically larger and therefore less dense in the sub-Neptune mass range than the RV planets.

There are biases in both techniques that contribute to this separation. In RV, for a given planetary size, there is a detection bias towards higher masses. TTVs, however, are strongly biased towards larger planets with deeper transit signals in a given mass range. Larger planets have more precisely measurable transit times and are the best candidates for TTV analysis. The bias is indirect, since the TTVs detected in a large planet are used to infer the masses of its neighbors, not itself. Nevertheless, in the complex TTV signals of systems with multi-planet interactions, like Kepler-79, the model as a whole benefits from the precisely measured transit times of the largest planet.

Another bias in the density distributions observed by each technique stems from the separation of the two samples shown in Figure 1. The low mass planets detected by RV all have high incident fluxes, and many of the low mass planets that are detectable are too hot to retain deep atmospheres (Lopez \& Fortney 2013a; Owen \& Jackson 2012). Hence there is a bias towards high densities in the RV sample. The TTV sample on the other hand, extends to distances where low-mass planets can retain deep atmospheres. However, it remains unknown whether or not the extreme low-density super-Earth mass planets are common, or whether they are limited to planets near the threshold of massloss over a small range of distances. 


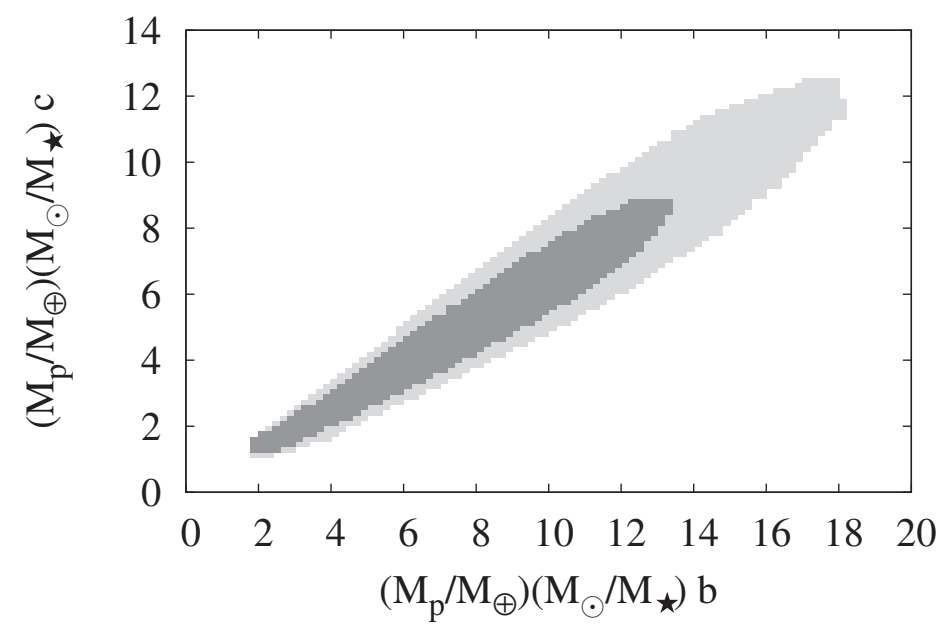

Figure 5. Joint posteriors for planetary masses relative to the host star for Kepler-29 b and c. $95.4 \%$ credible intervals are in light grey, $68.3 \%$ credible intervals are in dark grey. The dynamical masses are expressed relative to the host star, in approximate Earth-masses.

There may be additional detection biases in both RV and TTV based on planet formation and stellar properties. Most of the RV targets are cool stars, and RV surveys of the Kepler multi-planet systems have focused on size-limited surveys. TTV studies have focused on the most compact high multiplicity systems. Whether these planets truly represent all low-mass exoplanets within 1 AU remains to be seen.

Despite these biases, the RV and TTV samples overlap. This increases the scope of what we can study in multi-planet systems. In many cases, the mass posteriors from TTV analysis of planet pairs are strongly correlated, such that an additional constraint on either planetary mass from RV would enable both to be precisely characterized. An example is shown Figure 5, where the masses of Kepler-49 b and c are both well-constrained by TTVs and likely below the mass of Neptune. However, the ratio of the two masses is even better constrained, and thus additional constraints on the mass of the inner planet from RV would enable both planets to be further characterized.

\section{Precise Eccentricities}

In compact multi-planet systems, TTVs are particular sensitive to small changes in orbital eccentricity. Hence, where degeneracies between mass and eccentricity can be broken, precise eccentricities can be inferred (Deck \& Agol 2015), including in the regime where $e<0.1$. Lissauer et al. (2013) found upper limits on the eccentricities of Kepler-11 $\mathrm{d}$ and Kepler-11 e below $\sim 0.02$. Other exoplanets with precise limits on orbital eccentricities below $\sim 0.05$ include Kepler-30 b-d (Sanchis-Ojeda et al. 2012), Kepler-89 c-e Masuda et al. (2013), Kepler-79 b-e (Jontof-Hutter et al. 2014), Kepler-51 b-d (Masuda 2014), Kepler-88 b and c (Nesvorný et al. 2013), and Kepler-46 b (Nesvorný et al. 2012). However, there are few other examples of such precise constraints on orbital eccentricity from published TTV analyses, and it is unclear whether a large sample of precise eccentricities will come from the Kepler dataset. In some cases, precise individual eccentricities cannot be inferred from the transit times, even though relative eccentricities are well constrained. Jontof-Hutter et al. (2015) found that within the bounds of having well-constrained relative eccentricities, correlated posteriors in eccentricity vector 
components permit many solutions of high eccentricity. In such cases, the requirement of long-term stability cannot rule out the high eccentricity solutions, since the solutions are apsidally aligned and remain stable due to strong secular interactions.

Nevertheless, population statistics from the TTV sample can be used to infer eccentricity distributions. Using TTV phases and amplitudes for planet pairs near first-order mean motion resonance, Hadden \& Lithwick (2014) inferred an eccentricity distribution consistent with a Rayleigh distribution with a scale parameter $\sigma_{e}=0.018$. They found moderate evidence of higher eccentricities among smaller planets, although this could be due to larger timing errors on smaller planets and a TTV detection bias to high eccentricity for low mass planets.

\section{Stellar Parameters}

Planetary masses and radii cannot be characterized without precise constraints on the properties of the host, particularly the stellar radius. For the nearest planetary systems, interferometry directly constrains the stellar radius to the great precision (e.g., 55 Cancri, Gillon et al. 2012). Among targets in the Kepler field, asteroseismology detections on Kepler-10 (Batalha et al. 2011), Kepler-36 (Carter et al. 2012), Kepler-68 (Gilliland et al. 2013), and Kepler-93 (Dressing et al. 2015), have permitted uncertainties on stellar radius down to just $1-2 \%$. This remains the gold standard for targets in the Kepler field, although detections are limited to evolved stars and the brightest dwarf stars.

Transit light curves provide an independent constraint on the bulk density of the star, and hence its mass and radius (Seager \& Mallén-Ornelas 2003; Kipping 2010). The constraint depends on orbital period, transit depth, the duration of ingress or egress and the total transit duration (all of which come directly from the light curve), as well as orbital eccentricity. In most cases if $e \lesssim 0.1$, one has no choice but to assume the orbits are circular, although this adds an error of $\sim e \sin \omega$ to the inferred stellar radius.

In select cases, precise constraints on eccentricity vectors from TTVs enables more precise stellar radii measurements. For both Kepler-11 and Kepler-79 (Lissauer et al. 2013; Jontof-Hutter et al. 2014), stellar and hence planetary radii were constrained to a precision of $\sim 2 \%$, enabling meaningful constraints on the planetary bulk densities.

\section{A mass-radius-flux trend for low-mass exoplanets}

To account for the effect of flux on the observed density of planets, we compare planet radii to worlds made of pure silicate rock of the same mass. Planets larger than a purely rocky world must retain some significant volume of volatiles in the form of ices and gases. However, planets that are larger and less dense than a world made of pure water ice must retain deep atmospheres of $\mathrm{H} / \mathrm{He}$.

Figure 6 highlights the effect of incident flux on the range of densities observed in low mass exoplanets. The wide range in bulk densities among super-Earth mass planets makes a simple mass-radius fit to the data sensitive to a small number of planets with wide scatter in radius. The transition from a wide range of densities at low incident flux to the sample of predominantly rocky hot super-Earths is consistent with atmospheric mass loss (Owen \& Wu 2013).

The majority of systems with transiting planets and anti-correlated TTVs have yet to be analyzed for detailed characterizations (Mazeh et al. 2013), and many of the hosts require additional data and modeling to further constrain their properties. Hence, the addition of more precise TTV mass characterizations on the mass-radius diagram is 

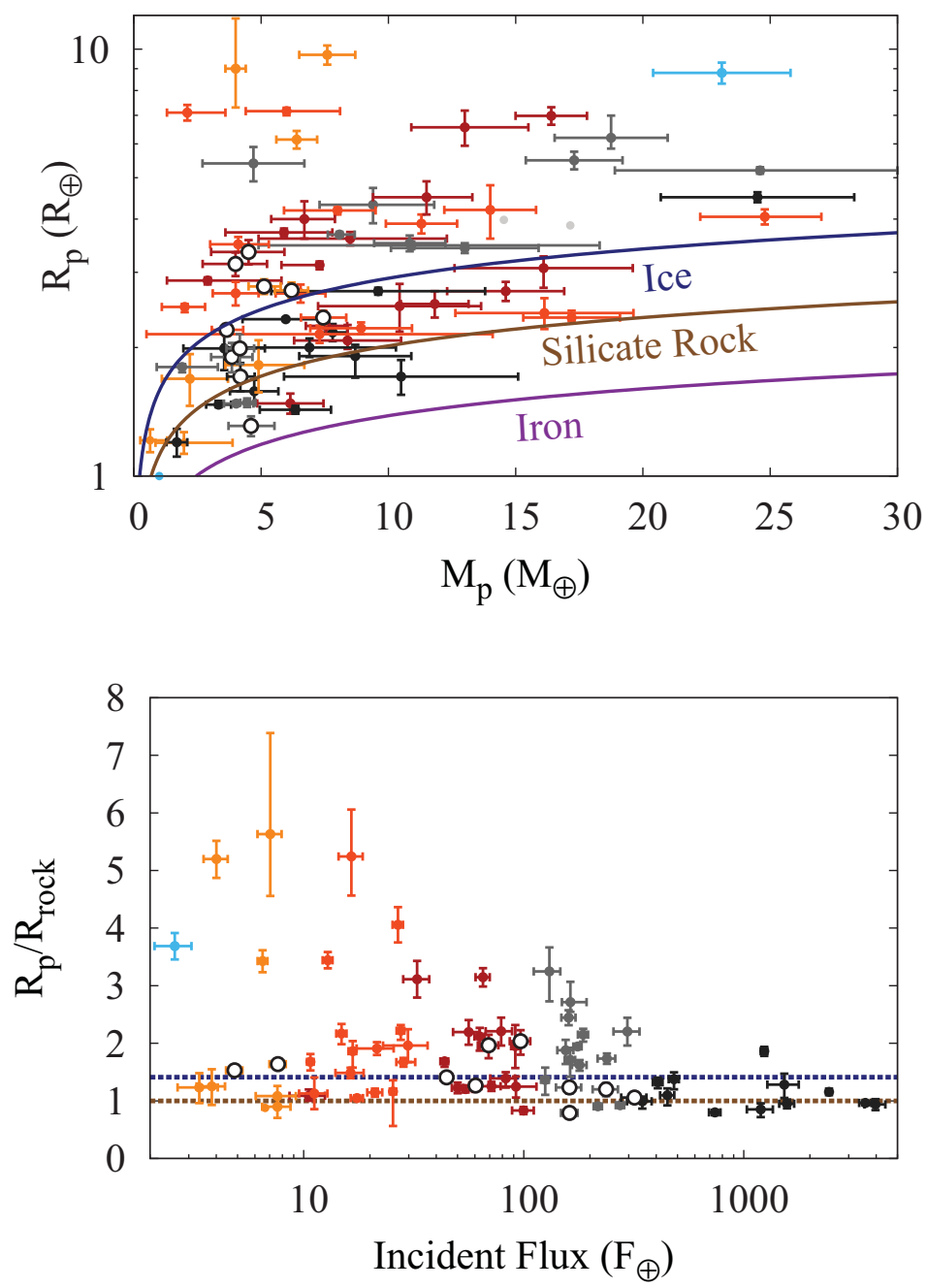

Figure 6. Mass-radius-flux diagram of planets less than $25 M_{\oplus}$. The colors in the top panel are coded by incident flux, which are illustrated in the lower panel. The lower panel compares planet radii to a planet made of pure silicate rock. Above the brown line, planets are less dense than rock and above the blue line, less dense than water. The range in planetary densities in this mass range is widest at low incident flux, while at high incident flux, most of the characterized planets are likely rocky. (Adapted from Jontof-Hutter et al. 2015, submitted)

certain. These will place additional constraints on mass-loss and formation scenarios for a wide range of stellar types and system architectures.

\section{Non-transiting planets}

Measuring planetary densities is only possible with TTVs if interacting planets are both transiting. TTVs caused by non-transiting perturbers enables the detection of additional planets, often orbiting further out than the known transiting planet. There are several single transiting systems in the Kepler dataset with significant TTVs likely caused by non-transiting planets (Mazeh et al. 2013). 


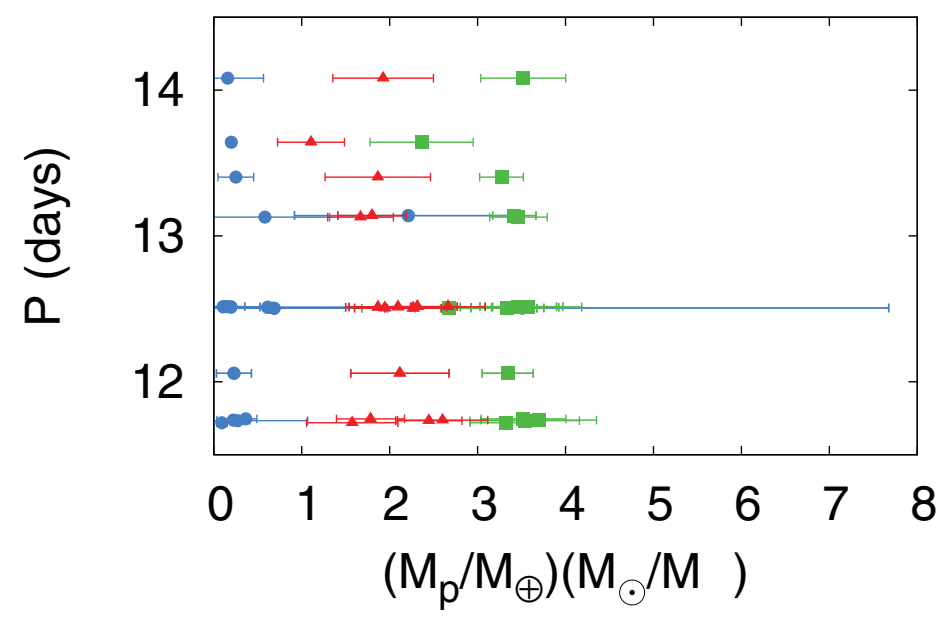

Figure 7. Solutions from a three-planet model for the TTVs of Kepler-50. The two known planets, Kepler-50 b (red triangles) and Kepler-50 c (green squares) have their masses compared to a non-transiting perturber (blue circles) for many potential orbital periods of the perturber. Although the parameters of the non-transiting planet are poorly constrained, the masses of the two transiting super-Earths are very well-constrained.

The strongest TTV detections permit a unique solution for the orbital period of a non-transiting perturber. Nesvorný et al. (2012) found a unique solution for a perturber of Kepler-46 b, attributable to a Saturn-mass perturber orbiting farther out. In another example, Nesvorný et al. (2013) analyzed the TTVs of Kepler-88 b (KOI-142.01) to predict the mass and orbital period of a jovian-mass planet that was soon confirmed with radial velocity observations (Barros et al. 2014).

For lower signal-to-noise TTVs (and lower mass planets), a detected periodicity can be attributed to a perturber at multiple locations near different resonances, with weak constraints on the perturbing mass. Kepler-50 has two transiting super-Earth sized planets with strongly detected TTVs (Steffen et al. 2013). While a two-planet model fails to fit the TTVs, a three planet model permits a close fit. In this case, there is no unique solution for the orbit period, phase or mass of the third planet. However, there are still useful constraints on the transiting planets. Figure 7 shows the potential orbital period of a third planet and the inferred planetary masses for all three objects for a range of solutions that fit the transit timing data and are stable for at least $100 \mathrm{kyr}$. Despite the poor constraints on the non-transiting perturber, these preliminary results imply that robust masses for the two transiting planets may be inferred.

\section{Conclusion}

Transit timing studies have yielded precise masses, precise orbital eccentricities and have even improved stellar parameters for transiting exoplanet systems, complementing the sample of transiting exoplanets confirmed via RV. In probing low-mass planets beyond the range of RV, TTVs have pushed mass characterization towards the regime of the terrestrial planets of the Solar System. The same studies however, have shown that terrestrial planets like our own are not typical of low mass planets within 1 AU. Rather super-Earth mass planets show a diversity in composition from rocky to Saturn-size gaseous worlds on the threshold of atmospheric mass loss. This fundamental result is one of many contributions to Kepler's legacy. Exoplanet science will continue to benefit 
from the contribution of TTVs into era of TESS and PLATO, to detect, confirm and characterize transiting planets and the architectures of multi-planet systems.

\section{References}

Agol, E. \& Deck, K. 2015, ArXiv e-prints

Agol, E., Steffen, J., Sari, R., \& Clarkson, W. 2005, MNRAS, 359, 567

Barros, S. C. C., et al. 2014, A\&A, 561, L1

Batalha, N. M., et al. 2011, ApJ, 729, 27

Borucki, W., et al. 2008, in IAU Symposium, Vol. 249, IAU Symposium, ed. Y.-S. Sun, S. FerrazMello, \& J.-L. Zhou, 17-24

Carter, J. A., et al. 2012, Science, 337, 556

Cochran, W. D., et al. 2011, ApJS, 197, 7

Deck, K. M. \& Agol, E. 2015, ApJ, 802, 116

Dressing, C. D., et al. 2015, ApJ, 800, 135

Dumusque, X., et al. 2014, ApJ, 789, 154

Fabrycky, D. C., et al. 2012, ApJ, 750, 114

-. 2014, ApJ, 790, 146

Ford, E. B., et al. 2011, ApJS, 197, 2

-. 2012a, ApJ, 750, 113

-. 2012b, ApJ, 756, 185

Fortney, J. J., Marley, M. S., \& Barnes, J. W. 2007, ApJ, 659, 1661

Gautier, III, T. N., et al. 2012, ApJ, 749, 15

Gilliland, R. L., et al. 2013, ApJ, 766, 40

Gillon, M., et al. 2012, A\&A, 539, A28

Hadden, S. \& Lithwick, Y. 2014, ApJ, 787, 80

Haywood, R. D., et al. 2014, MNRAS, 443, 2517

Holman, M. J. \& Murray, N. W. 2005, Science, 307, 1288

Holman, M. J., et al. 2010, Science, 330, 51

Howard, A. W., et al. 2013, Nature, 503, 381

Jontof-Hutter, D., Lissauer, J. J., Rowe, J. F., \& Fabrycky, D. C. 2014, ApJ, 785, 15

-. 2015, Nature, 785, 15

-. 2015, ApJ submitted

Kipping, D. M. 2010, MNRAS, 407, 301

Kipping, D. M., Nesvorný, D., Buchhave, L. A., Hartman, J., Bakos, G. Á., \& Schmitt, A. R. 2014, ApJ, 784, 28

Lissauer, J. J., et al. 2011a, Nature, 470, 53

-. 2011b, ApJS, 197, 8

-. 2013, ApJ, 770, 131

-. 2014, ApJ, 784, 44

Lithwick, Y. \& Wu, Y. 2012, ApJL, 756, L11

Lithwick, Y., Xie, J., \& Wu, Y. 2012, ApJ, 761, 122

Lopez, E. D., \& Fortney, J. J. 2013a, ApJ, 776, 2

Marcy, G. W., et al. 2014, ApJS, 210, 20

Masuda, K. 2014, ApJ, 783, 53

Masuda, K., Hirano, T., Taruya, A., Nagasawa, M., \& Suto, Y. 2013, ApJ, 778, 185

Mazeh, T., et al. 2013, ApJS, 208, 16

Nesvorný, D. \& Morbidelli, A. 2008, ApJ, 688, 636

Nesvorný, D., Kipping, D. M., Buchhave, L. A., Bakos, G. Á., Hartman, J., \& Schmitt, A. R. 2012, Science, 336, 1133

Nesvorný, D., Kipping, D., Terrell, D., Hartman, J., Bakos, G. Á., \& Buchhave, L. A. 2013, ApJ, 777, 3

Nesvorný, D. \& Vokrouhlický, D. 2014, ApJ, 790, 58

Ofir, A., Dreizler, S., Zechmeister, M., \& Husser, T.-O. 2014, A\&AA, 561, A103 
Owen, J. E. \& Jackson, A. P. 2012, MNRAS, 425, 2931

Owen, J. E. \& Wu, Y. 2013, ApJ, 775, 105

Pepe, F., et al. 2013, Nature, 503, 377

Ragozzine, D. \& Holman, M. J. 2010, ArXiv e-prints

Rogers, L. A. 2015, ApJ, 801, 41

Rowe, J. F., et al. 2014, ApJ, 784, 45

Sanchis-Ojeda, R., et al. 2012, Nature, 487, 449

Seager, S. \& Mallén-Ornelas, G. 2003, ApJ, 585, 1038

Steffen, J. H., et al. 2012a, MNRAS, 421, 2342

-. 2013, MNRAS, 428, 1077

Weiss, L. M., et al. 2013, ApJ, 768, 14

Wu, Y. \& Lithwick, Y. 2013, ApJ, 772, 74

Xie, J.-W. 2013, ApJS, 208, 22 